\title{
O IMPACTO DA PANDEMIA POR COVID-19 NA SAÚDE MENTAL DOS PROFISSIONAIS DA SAÚDE: REVISÃO INTEGRATIVA
}

\section{THE COVID-19 PANDEMICI'S IMPACT ON THE MENTAL HEALTH OF HEALTH PERSONNELS: INTEGRATIVE REVIEW}

\author{
Gabriela Duarte Bezerra ${ }^{1}$ Aline Sampaio Rolim de Sena ${ }^{1}$ 'Sara Teixeira Braga ${ }^{\text {* }}$ \\ Marcia Eduarda Nascimento dos Santos ${ }^{1 *}$ Lorena Farias Rodrigues Correia $^{1 *}$ Kyohana $^{*}$ \\ Matos de Freitas Clementino ${ }^{1 *}$ Yasmin Ventura Andrade Carneiro ${ }^{1 *}$ Woneska $^{*}$ \\ Rodrigues Pinheiro ${ }^{1}$
}

\begin{abstract}
RESUMO
Identificar os fatores que impactam na saúde mental dos profissionais da saúde no enfrentamento da COVID-19. Trata-se de uma revisão integrativa da literatura, realizado nas bases de dados: LILACS, MEDLINE, BDENF, PubMed, SCOPUS e COCHRANE. Foi utilizado para busca o Portal de Periódicos da CAPES e a Biblioteca Virtual da Saúde (BVS), através da ferramenta de busca avançada. A amostra foi composta por 15 artigos. Os estudos reforçam que as principais implicações na saúde mental dos profissionais relacionam-se principalmente à depressão, insônia, ansiedade, angústia, Transtorno de Estresse Pós-Traumático-TEPT, distúrbios do sono, síndrome de Burnout, Transtorno Compulsivo Obsessivo- TOC, exaustão, além de níveis mais baixos de satisfação no trabalho. Os estudos considerados para esta pesquisa refletem diretamente na saúde física das pessoas e no combate ao agente patogênico que são os focos primários de atenção de gestores e profissionais da saúde. Os estudos trazem como foco principal o estresse e a ansiedade que interferem na saúde desses trabalhadores. Conclui-se que, os profissionais da saúde que atuam na linha de frente contra a pandemia da COVID-19, nunca se encontraram em situações tão estressantes. Medidas para mantê-los saudáveis devem ser realizadas, desde a melhoria das condições de trabalho até obter recursos para prestação da assistência, treinamentos adequados, otimização das exaustivas jornadas de trabalho e meio propício ao descanso dos profissionais.
\end{abstract}

Palavra chave: Profissionais da saúde; Coronavírus; Saúde mental; Trabalho.

\begin{abstract}
Identify the factors that impact the mental health of health care workers in coping with COVID-19. It is an integrative literature review, done in the databases: LILACS, MEDLINE, BDENF, PubMed, SCOPUS and COCHRANE. It was used to research the CAPES Journals Portal and the Virtual Health Library (BVS), using the advanced research. The sample consisted of 15 articles. The studies reinforce that the main results in the mental health of professionals are mainly related to depression, insomnia, anxiety, anguish, Post-Traumatic Stress Disorder-PTSD, sleep disorders, Burnout syndrome, Obsessive Compulsive Disorder- OCD, exhaustion, besides lower levels of job satisfaction. The studies considered for this research directly reflect on the physical health of people and the fight against the pathogen that are the primary focus to attention for managers and health professionals. The studies focus on stress and anxiety that interfere with health care workers. It is concluded that health professionals who work on the front line against the COVID-19 pandemic, never referred so stressful situations. Measures to keep them healthy must be done, from the improvement of working conditions to get resources to provide assistance, adequate training, optimization of exhaustive workdays and a favorable environment for the professional's rest.
\end{abstract}

Keywords: Health Personnel, Mental health, Coronavirus and Work.

\footnotetext{
${ }^{1}$ Universidade Regional do Cariri. Crato-Ceará, Brasil.
} 


\section{INTRODUÇÃO}

O coronavírus é uma patologia responsável por causar síndromes respiratórias e gastrointestinais, tratando-se de um RNA vírus da ordem Nidovirales da família Coronaviridae. Quando infectam humanos, sua sintomatologia é do resfriado comum, podem levar a infecções graves principalmente em grupos de risco, idosos e crianças. Em 11 de março de 2020 a Organização Mundial de Saúde (OMS) caracteriza como estado de pandemia o surto mundial da doença causada pelo novo coronavírus denominada como COVID-19, que trouxe aos serviços de saúde um cenário de ações em assistência e segurança para à comunidade e profissionais envolvidos nos cuidados à população ${ }^{(1-2)}$.

A transmissão é favorecida através do contato próximo e desprotegida com materiais contaminados que servem de veículo para transmissão, assim como por secreções ou excreções de um indivíduo infectado, principalmente por meio de gotículas respiratórias. Pode acontecer, dependendo da concentração de partículas virais no ambiente, tendo sido demonstrada viabilidade viral em dispersão aerossol por três horas ou mais. Outros fluidos corporais não estão claramente implicados na transmissão do novo coronavírus, mas considera-se que há riscos de adoecimento caso haja contato desprotegido com sangue, fezes, vômitos e urina ${ }^{(1)}$.
O período médio de incubação é, em média, de 5.2 dias, com intervalo que pode chegar até 12.5 dias, há indícios que a transmissão também possa acontecer através de indivíduos assintomáticos. A maior parte dos casos de COVID-19 cursam com sintomatologia clínica leve, febre, tosse e dispnéia, podendo haver mialgia, confusão, cefaleia, dor de garganta, rinorreia, dor no peito, diarréia, náusea e vômito. Os casos mais graves evoluem para Síndrome Respiratória Aguda Grave (SRAG), lesão cardíaca aguda e lesões secundárias. Em relação a sua letalidade, está associada à idade mais avançada, comorbidades como distúrbios respiratórios crônicos, doenças cardiovasculares, diabetes mellitus e agravos oncológicos $^{(3,2)}$.

Mundialmente, até 20 de abril de 2020, foram confirmados 2.423 .470 casos de COVID-19 com 166.041 óbitos. Os Estados Unidos da América é o país com maior número de casos (764.265). O Brasil é o $11^{\circ}$ em número de infectados, onde contabilizou 40.581 casos e 2.575 óbitos, a maior parte desses casos concentrou-se na região Sudeste (21.836), região Nordeste (10.088) e Sul (2.921). A partir da correlação da incidência de COVID-19, os indicadores sociais, sociodemográficos e de saúde, observou que as capitais com maior desenvolvimento econômico, populacional foram as mais acometidas pelo coronavírus, 
isso deve-se, principalmente, pelo maior fluxo de viajantes ${ }^{(4,1)}$.

Pela ausência, no momento, de vacinas e tratamentos específicos para a doença, às experiências mundiais apontam para necessidade de controle na progressão da curva epidemiológica através do isolamento físico social e medidas no âmbito hospitalar, reduzindo a transmissão na comunidade, necessidade de suporte ventilatório e internação em unidades de terapia intensiva em curto espaço de tempo. Tudo isso implica na saúde mental da população, somado com rápida disseminação do vírus, tempo de duração e controle, esses fatores são agravados ainda mais devido à grande quantidade de informações falsas que vêm sendo compartilhadas ${ }^{(5,6)}$.

Os profissionais da saúde diariamente enfrentam condições de trabalho instáveis, em um ambiente marcado pela falta de segurança, infraestrutura inadequada e pelos riscos nele presentes. Isto influi em níveis altos de desgaste profissional, adoecimento físico e psicológico, má qualidade de vida e assistência à saúde. Essas situações são mais comuns aos profissionais atuantes na linha de frente de combate a COVID-19, devido às longas horas de trabalho, preocupação com o estado de saúde dos pacientes e com a escassez de Equipamentos de Proteção Individual (EPI), principalmente em países com recursos $\operatorname{limitados}^{(7,8-6)}$.
Os altos níveis de estresse apresentam uma séria ameaça à saúde mental dos profissionais, elevando as taxas de ansiedade, depressão, transtorno de estresse pós-traumático (TEPT) e comportamentos sociais negativos o que pode implicar na eficácia da jornada de trabalho dos profissionais da saúde ${ }^{(9,10)}$. Por conseguinte, o presente estudo tem como objetivo identificar os fatores que impactam na saúde mental dos profissionais da saúde no enfrentamento da COVID-19.

\section{MÉTODO}

O método de síntese do conhecimento adotado foi uma revisão integrativa da literatura, que permite a formulação de conclusões gerais a respeito de determinada área do conhecimento, mediante uma síntese de estudos publicados na perspectiva em estudo ${ }^{(11)}$.

$\mathrm{O}$ estudo compreendeu as seguintes etapas: I) identificação do tema e seleção da hipótese ou questão de pesquisa para a elaboração da revisão integrativa; II) estabelecimento de critérios para inclusão e exclusão de estudos/amostragem ou busca na literatura; III) definição das informações a serem extraídas dos estudos selecionados/categorização dos estudos; IV) avaliação dos estudos incluídos na revisão integrativa; V) interpretação dos resultados; 
VI) apresentação da revisão/síntese do conhecimento $^{(12)}$.

A questão norteadora da presente revisão foi: Quais os principais fatores que impactam na saúde mental dos profissionais da saúde no enfrentamento da COVID-19? Para encontrar respostas adequadas à pergunta de pesquisa e com vistas a uma melhor definição da população e do contexto, variáveis de interesse e de resultados optou-se pela estratégia Population, Variables and Outcomes (PVO) para busca dos artigos descritos no Quadro 1.

Quadro 1- Descritores de assunto localizados no DeCS para os componentes da pergunta de pesquisa segunda a estratégia PVO. Crato, CE, Brasil, 2020.

\begin{tabular}{|l|l|l|l|}
\hline Itens da estratégia & Componentes & $\begin{array}{l}\text { Descritores do } \\
\text { assunto (DeCS) }\end{array}$ & $\begin{array}{l}\text { Descritores do assunto } \\
\text { (MeSH) }\end{array}$ \\
\hline Population & Profissionais & $\begin{array}{l}\text { Profissionais da } \\
\text { saúde }\end{array}$ & Health Personnel \\
\hline Variables & Saúde & Saúde mental & Mental health \\
\hline Outcomes & Coronavírus & Pandemia & Pandemic \\
\hline Outcomes & Fatores que impactam & Impactos na Saúde & Impacts on Health \\
\hline
\end{tabular}

Elaborado pelos autores, 2020.

A partir da pergunta de pesquisa e do objetivo, foram definidos os Descritores em Ciências da Saúde (DeCs) e os Medical Subject Headings (MeSH), eles têm como objetivo uniformizar a linguagem dos artigos indexados nas bases de dados. Foram utilizados os DesCs: Profissionais da saúde, Saúde mental, Pandemia, Impactos na Saúde. Quanto aos MeSH, foram empregados: Health Personnel, Mental health, Pandemic, Impacts on Health com o operador booleano AND.

A coleta de dados estruturada aconteceu entre março e abril de 2020, com busca avançada, por permitir interligar descritores objetivando refinar a pesquisa, nas bases de dados: Literatura Latino-Americana e do Caribe em Ciências da Saúde (LILACS), Medical Literature Analysis and Retrieval System Online (MEDLINE) Banco de Dados em Enfermagem (BDENF), National Library of Medicine National Institutes of Health (PubMed), SciVerse (SCOPUS) e COCHRANE.

A busca foi realizada em pares e $o$ desempate para decidir a inclusão ou a exclusão de determinados estudos ficou 
como responsabilidade de um terceiro pesquisador.

O processo de seleção dos estudos foi realizado por meio da leitura minuciosa de títulos e resumos, seguindo os critérios de seleção previamente estabelecidos. Para a definição de critérios de inclusão levouse em consideração estudos primários, sem limite temporal. Optou-se por não definir um idioma para a busca dos artigos, objetivando reunir o maior número de publicações a respeito do tema em estudo.

Foram excluídos teses, dissertações, artigos não disponíveis na íntegra e estudos duplicados. Não foram considerados, também, estudos de casos. Para cada publicação excluída, foi avaliado e descrito o motivo de exclusão, sendo por fim separado os motivos em: estudos de outra natureza, fuga do tema proposto e artigos não disponíveis.

A busca dos estudos nas bases de dados resultou em 745 estudos. Para as bases MEDLINE, LILACS, e BDENF foram utilizadas as estratégias de busca: "Profissionais da saúde" AND "saúde mental” AND “pandemia”. Na MEDLINE após a utilização dos descritores, obtiveram-se 30 estudos que, após a aplicação dos critérios de inclusão, exclusão e a leitura dos artigos, foram selecionados oito estudos como resultado final. Na LILACS, foram identificados 10 estudos que, após todas as análises, foi selecionado cinco artigos e na BDENF teve 15 estudos, que após as análises restaram cinco artigos selecionados.

Para as bases SCOPUS, PUBMED e COCHRANE foram utilizadas as seguintes estratégias: "Health personnel" AND "mental health" AND "pandemic" foram encontrados 394 estudos na SCOPUS, 236 na PUBMED e 60 na COCHRANE. Após aplicação dos critérios de inclusão do estudo e análise dos títulos e resumos, foram selecionados para a leitura oito artigos na SCOPUS e sete na PUBMED.

Em resumo, foram identificados 745 estudos, dos quais após a leitura dos títulos e resumos foram rejeitados 634 estudos, que não se relacionavam a pesquisa com base nos critérios de inclusão e de exclusão, restando 111 artigos. Após a realização da leitura na íntegra selecionouse 77 artigos, destes oito eram estudos repetidos, cinco estudos incompletos, vinte e sete estudos de outra natureza e quatro fugia do tema proposto, restando 33 estudos com critérios de elegibilidade. Destes 22 artigos foram incluídos para avaliação, culminando em 15 artigos considerados relevantes para a revisão integrativa (FIGURA 1).

O instrumento Preferred Reporting Items for Systematic Rewiew and Meta-Analyses (PRISMA) demonstra 
o processo de busca e seleção dos estudos, conforme Figura 1:

Figura 1 - Fluxograma do processo de busca e seleção dos artigos por meio de cruzamentos. Crato, Ceará, 2020.
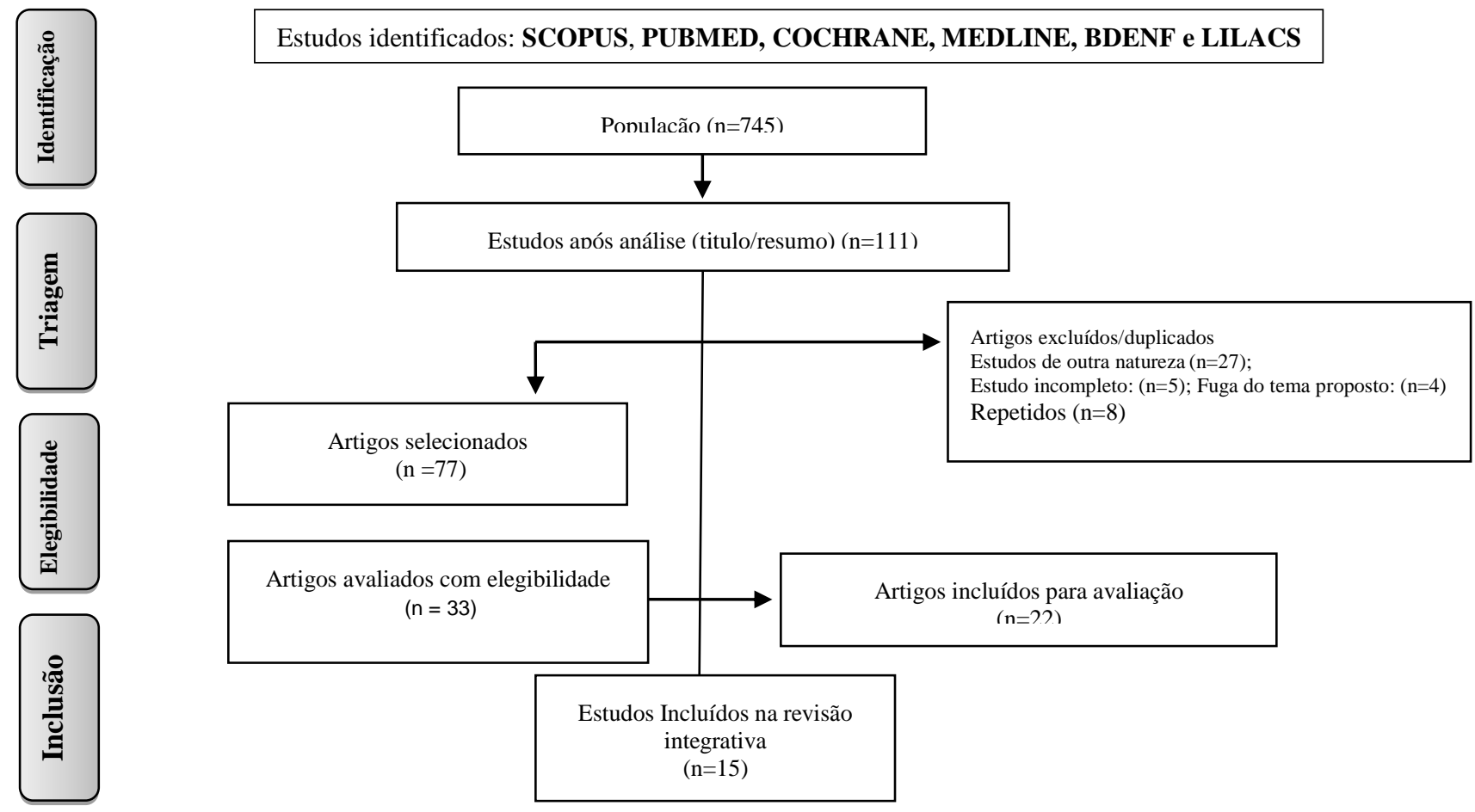

Elaborado pelos autores, 2020.

A análise dos estudos levantados foi realizada em pares por meio de um instrumento validado, proposto por Ursi e Galvão, adaptado para atender ao objetivo dessa pesquisa. $\mathrm{O}$ instrumento contem variáveis que respondem a questão norteadora do estudo, de modo que os tópicos de interesse foram: título do artigo, autores, ano de publicação, local de estudo, idioma, periódico, população/amostra, nível de evidência (NE).

Realizou-se a categorização dos estudos que compuseram a amostra de acordo com a classificação hierárquica das evidências para a avaliação de pesquisas ou outras fontes de informação. A qualidade das evidências é classificada em VII níveis ${ }^{(13)}$ : NEI, as evidências são provenientes de revisão sistemática ou metanálise de todos relevantes ensaios clínicos randomizados controlados ou oriundos de diretrizes clínicas baseadas em revisões sistemáticas de ensaios clínicos randomizados controlados; NEII, evidências derivadas de pelo menos um ensaio clínico randomizado controlado bem delineado; NEIII, evidências obtidas de ensaios clínicos bem delineados sem randomização; NEIV, evidências provenientes de estudos de coorte e de 
caso-controle bem delineados; NEV, evidências originárias de revisão derivadas de um único estudo descritivo ou qualitativo; NEVII, evidências oriundas de opinião de autoridades e/ou relatório de comitês de especialistas. sistemática de estudos descritivos e qualitativos; NEVI, evidências

\section{RESULTADOS}

A amostra desta revisão foi composta por quinze estudos que tratam dos principais fatores que impactam na saúde mental dos profissionais da saúde no enfrentamento ao coronavírus.

Quadro 2- Caracterização da produção científica sobre os fatores que impactam na saúde mental dos profissionais da saúde no enfrentamento da Covid-19.

\begin{tabular}{|c|c|c|c|c|c|c|}
\hline Título & Autor & $\begin{array}{l}\text { Ano de } \\
\text { Public } \\
\text { ação }\end{array}$ & $\begin{array}{l}\text { Local } \\
\text { de } \\
\text { Estudo } \\
\end{array}$ & $\begin{array}{l}\text { Idioma/ } \\
\text { Periódico }\end{array}$ & $\begin{array}{l}\text { População/a } \\
\text { mostra }\end{array}$ & $\mathbf{N E}$ \\
\hline $\begin{array}{l}\text { Factors Associated With } \\
\text { Mental Health Outcomes } \\
\text { Among Health Care } \\
\text { Workers Exposed to } \\
\text { Coronavirus Disease } 2019\end{array}$ & $\begin{array}{l}\text { JIANB } \\
\text { O, et al. }\end{array}$ & 2020 & China & $\begin{array}{l}\text { Inglês, } \\
\text { JAMA } \\
\text { Network } \\
\text { Open }\end{array}$ & $\begin{array}{l}\text { Médicos e } \\
\text { Enfermeiros. }\end{array}$ & VI \\
\hline $\begin{array}{l}\text { A saúde mental da } \\
\text { enfermagem no } \\
\text { enfrentamento da COVID- } \\
19 \text { em um hospital } \\
\text { universitário regional }\end{array}$ & $\begin{array}{l}\text { DAL'B } \\
\text { OSCO, } \\
\text { et al. }\end{array}$ & 2020 & Paraná & $\begin{array}{l}\text { Português, } \\
\text { Rev } \\
\text { Brasileira } \\
\text { de } \\
\text { Enfermage } \\
\text { m } \\
\end{array}$ & $\begin{array}{l}\text { Profissionais } \\
\text { de } \\
\text { Enfermagem. }\end{array}$ & VI \\
\hline $\begin{array}{l}\text { Sleep disturbances among } \\
\text { medical workers during } \\
\text { the outbreak of COVID- } \\
2019 \text {. }\end{array}$ & $\begin{array}{l}\text { WANG } \\
\text {, et al. }\end{array}$ & 2020 & Wuhan & $\begin{array}{l}\text { Inglês, } \\
\text { Occupatio } \\
\text { nal } \\
\text { Medicine. }\end{array}$ & Médicos & IV \\
\hline $\begin{array}{l}\text { The impact of the } \\
\text { COVID-19 pandemic on } \\
\text { the mental health of } \\
\text { healthcare professionals. }\end{array}$ & $\begin{array}{l}\text { ORNN } \\
\text { EL, et } \\
\text { al. }\end{array}$ & 2020 & $\begin{array}{l}\text { Porto } \\
\text { Alegre }\end{array}$ & $\begin{array}{l}\text { Inglês, } \\
\text { Cadernos } \\
\text { de Saúde } \\
\text { Pública. }\end{array}$ & \begin{tabular}{ll}
\multicolumn{2}{l}{ Profissionais } \\
da saúde da \\
linha & de \\
frente. &
\end{tabular} & VI \\
\hline $\begin{array}{l}\text { The effect of Coronavirus } \\
\text { (COVID-19) on Breasth } \\
\text { Cancer Teamwork: A } \\
\text { multicentric survey }\end{array}$ & $\begin{array}{l}\text { VANN } \\
\text { I et al. }\end{array}$ & 2020 & Itália & $\begin{array}{l}\text { Inglês, In } \\
\text { vivo }\end{array}$ & $\begin{array}{l}\text { Profissionais } \\
\text { da Saúde. }\end{array}$ & IV \\
\hline $\begin{array}{l}\text { At the height of the storm: } \\
\text { Healthcare staff's health } \\
\text { conditions and job } \\
\text { satisfaction and their } \\
\text { associated predictors } \\
\text { during the epidemic peak } \\
\text { of COVID-19 }\end{array}$ & $\begin{array}{l}\text { ZHAN } \\
\text { G, et al. }\end{array}$ & 2020 & China & $\begin{array}{l}\text { Ingles, } \\
\text { Rain } \\
\text { Behavior } \\
\text { and } \\
\text { Immunity. }\end{array}$ & $\begin{array}{l}\text { Médicos, } \\
\text { Enfermeiros, } \\
\text { Radiologistas } \\
\text { e Técnicos. }\end{array}$ & VI \\
\hline $\begin{array}{l}\text { Psychosocial burden of } \\
\text { healthcare professionals in } \\
\text { times of COVID-19 - a } \\
\text { survey conducted at the } \\
\text { University Hospital } \\
\text { Augsburg. }\end{array}$ & $\begin{array}{l}\text { ZERBI } \\
\text { NI, et } \\
\text { al. }\end{array}$ & 2020 & $\begin{array}{l}\text { Augsbu } \\
\text { rg } \\
\text { (Alema } \\
\text { nha), }\end{array}$ & $\begin{array}{l}\text { Inglês, } \\
\text { German } \\
\text { Medical } \\
\text { Science. }\end{array}$ & Enfermeiros. & VI \\
\hline
\end{tabular}




\begin{tabular}{|c|c|c|c|c|c|c|}
\hline $\begin{array}{l}\text { Mental Health and } \\
\text { Psychosocial Problems of } \\
\text { Medical Health } \\
\text { Workersduring the COVID- } \\
\text { 19 Epidemic in China }\end{array}$ & $\begin{array}{l}\text { ZHANG, et } \\
\text { al. }\end{array}$ & 2020 & China & $\begin{array}{l}\text { Inglês, } \\
\text { Psychother } \\
\text { Psychosom. }\end{array}$ & $\begin{array}{l}\text { Profissiona } \\
\text { is da saúde } \\
\text { médicos e } \\
\text { não } \\
\text { médicos. }\end{array}$ & VI \\
\hline $\begin{array}{l}\text { Provider Burnout and Fatigue } \\
\text { During the COVID-19 } \\
\text { Pandemic: Lessons Learned } \\
\text { From a High-Volume } \\
\text { Intensive Care Unit }\end{array}$ & $\begin{array}{l}\text { SASANGO } \\
\text { HAR, et al. }\end{array}$ & 2020 & Texas & $\begin{array}{l}\text { Inglês, } \\
\text { Anesthesia \& } \\
\text { Analgesia }\end{array}$ & $\begin{array}{l}\text { Profissiona } \\
\text { is da saúde } \\
\text { na linha de } \\
\text { frente. }\end{array}$ & V \\
\hline $\begin{array}{l}\text { Posttraumatic } \\
\text { symptoms of health care } \\
\text { workers during the corona } \\
\text { virus disease } 2019\end{array}$ & $\begin{array}{l}\text { QIANLAN, } \\
\text { et al. }\end{array}$ & 2020 & China & $\begin{array}{l}\text { Inglês, Clin } \\
\text { Psychol } \\
\text { Psychother. }\end{array}$ & $\begin{array}{l}\text { Profissiona } \\
\text { is da saúde } \\
\text { na linha de } \\
\text { frente. }\end{array}$ & VI \\
\hline $\begin{array}{l}\text { Psychological impact of } 2019 \\
\text { novel coronavirus (2019- } \\
\text { nCoV) outbreak in health } \\
\text { workers in China. }\end{array}$ & SUN, et al. & 2020 & China & $\begin{array}{l}\text { Ingles, } \\
\text { Epidemiology } \\
\text { and Infection. }\end{array}$ & $\begin{array}{l}\text { Profissiona } \\
\text { is da saúde } \\
\text { na linha de } \\
\text { frente. }\end{array}$ & VI \\
\hline $\begin{array}{l}\text { Unravelling potential severe } \\
\text { psychiatric repercussions on } \\
\text { healthcarer professionals } \\
\text { during the COVID-19 crisis. }\end{array}$ & $\begin{array}{l}\text { ANMELLA } \\
\text {, et al. }\end{array}$ & 2020 & $\begin{array}{l}\text { Barcelo } \\
\text { na }\end{array}$ & $\begin{array}{l}\text { Inglês, Journal } \\
\text { of Affective } \\
\text { Disorders. }\end{array}$ & $\begin{array}{l}\text { Profissiona } \\
\text { is da saúde } \\
\text { na linha de } \\
\text { frente. }\end{array}$ & IV \\
\hline $\begin{array}{l}\text { Mental Health Outcomes } \\
\text { Among Frontline and Second- } \\
\text { Line Health Care Workers } \\
\text { During the Coronavirus } \\
\text { Disease } 2019 \text { (COVID-19) } \\
\text { Pandemic in Italy }\end{array}$ & $\begin{array}{l}\text { ROSSI, et } \\
\text { al. }\end{array}$ & 2020 & $\begin{array}{l}\text { Roma } \\
\text { (Itália) }\end{array}$ & $\begin{array}{l}\text { Inglês, JAMA } \\
\text { Network } \\
\text { Open. }\end{array}$ & $\begin{array}{l}\text { Profissiona } \\
\text { is da saúde } \\
\text { na linha de } \\
\text { frente. }\end{array}$ & VI \\
\hline $\begin{array}{l}\text { Mitigating the psychological } \\
\text { effects of COVID-19 on } \\
\text { health care workers. }\end{array}$ & $\begin{array}{l}\text { PETER, et } \\
\text { al. }\end{array}$ & 2020 & $\begin{array}{l}\text { Toront } \\
\text { o }\end{array}$ & $\begin{array}{l}\text { Inglês, Mental } \\
\text { Health. }\end{array}$ & $\begin{array}{l}\text { Profissiona } \\
\text { is da saúde } \\
\text { na linha de } \\
\text { frente. }\end{array}$ & VI \\
\hline
\end{tabular}

Elaborado pelos autores, 2020.

Os estudos reforçam que as principais implicações na saúde mental dos profissionais relacionam-se principalmente à depressão, insônia, ansiedade, angústia, Transtorno de Estresse Pós-TraumáticoTEPT, distúrbios do sono, síndrome de Burnout, Transtorno Compulsivo Obsessivo- TOC, exaustão, além de níveis mais baixos de satisfação no trabalho ${ }^{(14,15,16,17,18,19,20,21,22)}$.

Profissionais da linha de frente demonstraram risco de desenvolver transtorno de estresse pós-traumático (TEPT), que persistiu mesmo após um período de ausência do trabalho ${ }^{(23)}$. Já os 
profissionais acima de 56 anos que associados ao estresse pós-traumático desencadeado pela epidemia de SARS em $2003^{(24)}$. Assim como a síndrome de Burnout, que também foi relatada por profissionais da saúde envolvidos na assistência a pacientes durante uma epidemia causada por outro tipo de coronavírus que ocorreu na Coréia em $2016^{(17)}$.

Foi identificado que os profissionais da saúde do sexo feminino, principalmente enfermeiras, foram mais vulneráveis aos TEPT e à níveis mais altos de ansiedade, além disso, apresentaram maiores índices de depressão e angústia $^{(14,19,17)}$. Vale ressaltar que outros profissionais também referiram os mesmos agravos, porém em menor quantidade quando comparados com as mulheres. Já os distúrbios do sono, foram altamente prevalentes entre profissionais que atuam na pediatria, isso ocorre devido a maior aproximação com os pacientes e prolongamento dos turnos de trabalho ${ }^{(16)}$.

Ademais, os estudos ressaltaram que os profissionais de enfermagem estão mais propensos a serem afetados psicologicamente, isso foi justificado pelo fato deles estarem atuando em um contato mais próximo dos pacientes com a COVID-19 e por possuírem uma carga de trabalho elevada, aumentando, vivenciaram o transtorno, foram consequentemente, seu tempo de permanência na unidade hospitalar ${ }^{(17,20)}$.

Os profissionais com suspeita de COVID-19 apresentaram maior depressão, ansiedade, angústia e menor satisfação no trabalho, relacionados ao medo da disseminação do vírus para familiares, amigos e sua equipe de saúde (19,25,28). Além do enfrentamento da discriminação social, gerada contra indivíduos que foram acometidos pela doença ${ }^{(26)}$.

Com foco na investigação dos fatores ocupacionais que podem ter comprometido a saúde mental dos profissionais durante o período de pandemia, alguns estudos relataram que os riscos laborais devido a quantidade reduzida de insumos e Equipamentos de Proteção Individual (EPI), treinamento insuficiente em relação ao enfrentamento da doença em questão, assim como, o prolongamento da carga horária e o contato direto com pacientes com COVID-19, foram as causas mais citadas pelos trabalhistas, como agravantes psicológicos $(15,19,20,22,26)$. O estresse no ambiente de trabalho é um fator que pode contribuir para a exaustão psíquica dos profissionais, já que ocorre um desgaste emocional e cansaço físico e mental ${ }^{(17,20)}$.

Fatores relacionados à vínculos familiares e de amizade também foram associados à flagelação da saúde mental da 
equipe de saúde, pois ter um colega falecido, hospitalizado ou em quarentena devido a COVID-19 estava associado ao familiares e amigos, essas preocupações dos profissionais da saúde acabam sendo elevadas, devido a rápida disponibilidade de informações e a desinformação na Internet mídias sociais ${ }^{(17,22,28)}$.

\section{DISCUSSÃO}

Os artigos selecionados trazem uma gama de fatores que impactam diretamente na saúde mental dos profissionais da saúde que estão à frente no combate à Covid-19. Essas pessoas lidam todos os dias com situações difíceis, como perca de pacientes, ausência de recursos materiais tais como equipamentos de proteção de uso individual, complexidades dos níveis de gravidade da doença, protocolos novos e uma sobrecarga maior de trabalho.

Os estudos trazem como focos principais o estresse e a ansiedade que interferem na saúde desses trabalhadores $^{(29,30)}$. Em um estudo realizado sobre psicologia hospitalar mostra que devido ao alto índice de contagio da Covid-19, falta de materiais hospitalares, vivências diretas com o sofrimento de pacientes e famílias, acarretam episódios de ansiedade, transtornos de estresse e psicossomático, desenvolvimento de $\mathrm{TEPT}^{(27)}$. Outrossim, uma das preocupações mais frequente foi em disseminar a infecção entre os depressão e vários outros transtornos psicológicos que afetam a saúde, qualidade de vida e trabalho desses profissionais da saúde ${ }^{(31)}$.

Em outro estudo transversal, realizado por região, coletou-se dados demográficos em relação a medidas de saúde mental, obtendo um total 1257 profissionais da saúde em 34 hospitais nos meses de janeiro a fevereiro de 2020, na China. A identificação dos fatores associados aos resultados de saúde mental presentes também foram: sintomas de depressão, ansiedade, insônia e angústia sendo avaliada pela a Escala de Transtorno de Ansiedade Generalizada ${ }^{(14)}$.

Paralelo a isto, encontram-se os relatos dos profissionais da saúde sobre as más condições de trabalho, jornadas exaustivas, falta de treinamentos para novos protocolos, carência de materiais de proteção tornando, assim, um ambiente de trabalho hostil e com isto afetando diretamente a saúde da equipe no combate ao Covid-19 ${ }^{(32)}$.

Algumas literaturas demonstram que existem três grandes aspectos no trabalho que impactam na saúde mental dos profissionais, a física, cognitiva e psíquica e se tratando de circunstancias pandêmicas, essas dimensões tendem a se 
desenvolver de forma negativa para a saúde dos profissionais devido a sua grande sobrecarga, ainda acrescenta-se a carga moral, sendo este um dos fatores mais difíceis para a equipe, pois precisam demandar e tomar decisões que vão implicar diretamente na vida desses pacientes, podendo gerar por parte desses profissionais sentimentos de medo, angustias, desconforto, ansiedade afetando na saúde mental ${ }^{(6)}$.

As altas taxas de saúde mental prejudicada refletem principalmente nos profissionais do sexo feminino, enfermeiras, que tinham entre 26 e 40 anos, casadas e trabalhavam em hospitais terciários com título técnico júnior, estas profissionais que estão na linha de frente lidando com pacientes com COVID-19 foram associadas a um maior risco de sintomas de depressão, ansiedade, insônia e angústia ${ }^{(14)}$.

Outrossim, a condição a que os profissionais da linha de frente estão expostos durante a pandemia, tem um grande impacto sobre a sua qualidade do sono, o qual está correlacionado aos sintomas de ansiedade e depressão, acentuados nesse momento. Como resultado, a eficiência do sono é reduzida, o que pode afetar todos os aspectos da saúde física, como os sistemas cardiovascular, endócrino e imunológico, podendo até mesmo causar sintomas irreversíveis. E, a longo prazo, o sono insuficiente pode ocasionar sintomas emocionais, que possibilita o aumento das barreiras para várias funções fisiológicas, como imunidade, aprendizado e memória, expondo esse profissional também à maiores chances de contrair a doença ${ }^{(33)}$.

Assim, os sintomas físicos ligados a COVID-19 podem ser apresentados de forma moderada a severa, destacando-se a ansiedade (28,8\%), depressão (16,5\%) e estresse $(8,1 \%)$ respectivamente. $\mathrm{O}$ mesmo estudo relata que as mulheres são as mais perceptíveis ao contágio a doença devido ao medo de infectarem suas famílias na volta para casa ${ }^{(34)}$.

As mulheres que trabalham na área da saúde são as mais acometidas mentalmente, pois o sexo feminino compõe grande parte do pessoal de saúde e são muitas vezes, as principais responsáveis pelo trabalho doméstico e cuidado com os filhos, tendo maiores chances de, quando aliada as demais circunstâncias as quais estão inseridas, terem sua saúde mental afetada ${ }^{(35)}$.

Além da exaustiva carga de trabalho, esses profissionais ainda sofrem com estigmas e medo pela sociedade e familiares, por manterem um contado direto com o vírus cotidianamente, acarretando assim fatores sentimentais como emoções mistas ou ambivalentes, estresse e até mesmo sentimento de culpa 
por não serem capazes de realizar tarefas normais de casa e do trabalho ou paternidade durante $\mathrm{o}$ percurso da pandemia. Desse modo, permanecer conectado com os entes queridos em meios tecnológicos, ter uma boa comunicação com sua equipe, são maneiras de manter contato e amenizar as angústias ${ }^{(36)}$.

Os desafios enfrentados pelos profissionais da saúde podem ser um gatilho para o desencadeamento ou a intensificação de sintomas de ansiedade, depressão e estresse ${ }^{(26)}$, especialmente quando se trata daqueles que trabalham na chamada "linha de frente", ou seja, um fator de risco independente para pior resultados de saúde mental em todas as dimensões de interesse ${ }^{(14)}$.

O estresse relacionado ao trabalho é uma causa potencial de preocupação para os profissionais da saúde. Sendo associada à ansiedade, incluindo múltiplas atividades clínicas, depressão em face da coexistência de inúmeras mortes, turnos de trabalho com as mais diversas incógnitas e demandas no tratamento de pacientes com COVID-19, resultando em um importante indicador de exaustão psíquica ${ }^{(29)}$.

Estudos demonstram que o estresse psicológico e a ansiedade podem ser um dos fatores de risco para maior morbidade mediada pela COVID-19 entre a equipe de saúde ${ }^{(30)}$.
O medo é descrito em um dos estudos como uma consequência da quarentena. O serviço de saúde, contudo, durante as emergências é destacado com diversas lacunas, tal como a falta de treinamentos para prestação de cuidados, sendo um fator a saúde mental destes profissionais. Em outro estudo, os profissionais da saúde tendem a tomar decisões impossíveis e trabalhar sob pressões extremas $^{(37,38)}$.

Estas decisões incluem alocar recursos escassos para pacientes igualmente carentes, como equilibrar suas próprias necessidades de saúde física e mental com as dos pacientes, alinhar seu desejo e dever aos pacientes com aqueles para familiares e amigos, prestar assistência para todos os pacientes gravemente doentes com recursos limitados ou inadequados. Estas decisões resultam em danos morais e em problemas de saúde mental ${ }^{(38)}$.

Outro ponto importante é o uso adequado de medidas de proteção, um tema complexo e que pode sofrer influência de diversos fatores. Pois, autores afirmam que a consciência de estar em risco constante influência o comportamento dos profissionais. Havendo a necessidade de um órgão maior e discussões de questões que envolvem a biossegurança no desenvolvimento das 
atividades laborais, com foco para a diminuição dos riscos ocupacionais ${ }^{(39)}$.

As precauções padrão (PP) são medidas de proteção que devem ser executadas pelos trabalhadores considerando que quaisquer fluídos corporais, podem conter agentes infectantes. A maioria dos profissionais da saúde que trabalham em unidades de isolamento e hospitais não recebe treinamentos suficientes para lidar em meio a uma pandemia, dentre estes fatores e a não adesão profissional individual resulta em riscos ocupacionais a saúde ${ }^{(37,39)}$.

Diante da quantidade de informações disponíveis e a frequência cada vez maior de atualizações, percebe-se a dificuldade do profissional de saúde em adaptar-se, tendo em vista a insuficiência de tempo disponível e preparo mental para absorvê-las. Assim, fornecer informações para esses profissionais, a partir de fontes oficiais, por meio de aplicativos, possibilitará minimizar e esclarecer notícias falsas e disseminação de ambas. Fornece-lhes maior segurança, contribuindo para a prevenção de sintomas psíquicos e gerenciamento do estresse $^{(40,41)}$.

Embora a pandemia da COVID19 tenha permeado o medo não apenas a níveis individuais, mas em estâncias sociais se tem a necessidade de implementação às devidas precauções de saúde mental junto com precauções de saúde física, estudos vem corrobora com assistência as esses profissionais, buscando estratégias de apoio psicológicos on-line em casa e de acesso fácil ${ }^{(42)}$

\section{CONCLUSÃO}

Conclui-se que, os profissionais da saúde que atuam na linha de frente em meio à pandemia do coronavírus, mais do que nunca, encontram-se em situações estressantes, o que acarreta uma sobrecarga maior de preocupação, trabalho, ansiedade e depressão diante de tantas mortes e longos turnos de trabalho.

Atrelado a essa grande carga de trabalho e ao grande número de profissionais e pacientes infectados, a exaustão psíquica e a falta de equipamentos essenciais para lutar contra essa epidemia, tem colocando-os em situações difíceis.

Esses profissionais que estão vivenciando essa pandemia estão mais propensos a desenvolver problemas psíquicos como a depressão. Tem-se encontrado em exaustão física, estão lidando com ansiedade, insônia, angústia e o medo de ao retornarem aos seus lares, prejudicarem os seus familiares. A perda de pacientes em uma escala tão grande 
como em meio a pandemia e a pressão que esses profissionais vêm sofrendo é o que mais traz danos a sua saúde.

Portanto, é preciso frisar que, assim como os pacientes, esses profissionais também possuem pessoas que os amam, para as quais precisam voltar com saúde física e mental. Estes necessitam de um olhar mais sensível voltado às suas necessidades físicas,

\section{REFERÊNCIAS}

1. Silva AWC, Cunha A, Alves GC, Corona RA, Dias CAG, Nassiri R; et al. Perfil epidemiológico e determinante social do COVID-19 em Macapá, Amapá, Amazônia, Brasil. Revista Científica Multidisciplinar Núcleo do Conhecimento [Internet]. 2020 [acesso em 07 mai 2020]; 04 (04): 05-27. Disponível em:https://www.nucleodoconheciment o.com.br/saude/covid-19-em-macapa

2. Gallasch $\mathrm{CH}$, Cunha ML, Pereira, LAS; Silva-Junior JS. Prevenção relacionada à exposição ocupacional do profissional de saúde no cenário de COVID-19. Revista Enfermagem Uerj [Internet]. Rio de Janeiro. 2020[acesso em 11 mai 2020]; 28 (28): 1-6, 2. Disponível em: https://www.e- mentais e espirituais. Medidas para mantêlos saudáveis devem ser realizadas, desde a melhoria das condições de trabalho até a disponibilidade de recursos para prestação da assistência, treinamentos adequados, otimização das exaustivas jornadas de trabalho e meio propício ao descanso dos profissionais.

publicacoes.uerj.br/index.php/enferma gemuerj/article/view/49596/33146

3. Ministério da Saúde (Brasil), Protocolo de Manejo Clínico para o Novo Coronavírus (2019-Cov2). Brasília (DF); 2020. Disponível em: https://portalarquivos2.saude.gov.br.

4. Ministério da Saúde (Brasil), Secretaria de Vigilância em Saúde. Boletim Epidemiológico 13 Centro de Operações de Emergências em Saúde Pública, Doença pelo Coronavírus 2019 (COE-COVID19). 2020. Disponível em:https://portalarquivos.saude.gov.br/ images/pdf/2020/April/21/BE13--Boletim-do-COE.pdf

5. Rafael RMR, Mercedes N, Carvalho MMB, David HMSL, Acioli S, FariaMGA. Epidemiologia, políticas públicas e pandemia de Covid-19: o 
que esperar no Brasil?: o que esperar no Brasil? Revista Enfermagem Uerj, Rio de Janeiro [Internet]. 2020 [acesso em 07 mai 2020]; 28 (28): 1-6,2. Disponível em: https://www.epublicacoes.uerj.br/index.php/enferma gemuerj/article/view/49570/33134

6. Rego S, Palácios M. Saúde mental dos trabalhadores de saúde em tempos de coronavírus. 2020 [acesso em: 24 de abril de 2020]. Disponível em https://www.arca.fiocruz.br/handle/icic $\mathrm{t} / 40659$

7. Assari S, Habibzadeh P. The COVID19 Emergency Response Should Include a Mental Health Component. Archives OfIranian Medicine [Internet]. 2020 [acesso em 11 mai 2020]; 23 (4): 281-282. Disponível em: http://www.aimjournal.ir/Article/aim15550

8. Machado MH, Santos MRS, Oliveira E, Wermelinger M, Vieira M, Lemos $\mathrm{W}$, et al. Condições de trabalho da enfermagem. Enfermagem em Foco [Internet]. 2016 [acessoem11 mai 2020]; 7, 63-76.Disponível em: http://revista.cofen.gov.br/index.php/en fermagem/article/view/695/305

9. Cosic K, Popovic S, Sarlija M, Kesedzic I. Impact of human disasters and covid-19 pandemic on mental health: potential of digital psychiatry: potential of digital psychiatry. Psychiatria Danubina [Internet]. 2020 [acesso em:07 mai 2020]abr 15 2020; 32 (1): 25-31. Disponível em: http://www.psychiatriadanubina.com/UserDocsImages/pdf/dn b_vol32_no1/dnb_vol32_no1_25.pdf

10. Ministério da saúde. Resolución Ministerial -180: Cuidado de la Salud Mental del personal de la salud em el contexto del covid-19 Lima, 2020 [acesso em: 12 mai 2020]; 36. Disponível em: http://docs.bvsalud.org/biblioref/2020/ 06/1099619/rm_363-2020-minsa.pdf 11. Souza MT, Silva MD, Carvalho R. Revisão integrativa: o que é e como fazer. Einstein [Internet]. 2010 [acesso em 12 mai 2020]; 8 (1): 102-106. Disponível em: https://www.scielo.br/pdf/eins/v8n1/pt _1679-4508-eins-8-1-0102.pdf

12. Mendes KDS, Silveira RCCP, Galvão CM. Revisão integrativa: método de pesquisa para a incorporação de evidências na saúde e na enfermagem. Texto \& Contexto Enfermagem, Florianópolis [Internet]. 2008 [acesso em 07 mai 2020]; 17(4):758-64. Disponível em: https://www.scielo.br/scielo.php?pid=s 0104- 
07072008000400018\&script=sci_abstr act\&tlng=pt.

13. Galvão CM. Níveis de Evidência. Acta Paul Enferm 2006; 19 (2): V.

14. Lai J, Ma S, Wang Y, Cai W, Hu J, Wei N, et al. Factors Associated With Mental Health Outcomes Among Health Care Workers Exposed to Coronavirus Disease 2019. China: JAMA Network [Internet] 2020 [acesso em 4 de Agosto 2020]; 3 (3), e203396. Disponível em: https://pesquisa.bvsalud.org/portal/reso urce/es./mdl-32389999.

15. Dal-Bosco EB, Floriano LSM, Skupien SV, Acaro G, Martins AR, Anselmo ACC. A saúde mental da enfermagem no enfrentamento da COVID-19 em um hospital universitário regional. Paraná: Rev Brasileira de Enfermagem [Internet] 2020 [acesso em 4 de Agosto 2020];73(2);e20200434. Disponível em:

https://www.scielo.br/scielo.php?script =sci_abstract\&amp;pid=S0034716720 20001400153\&amp;lng=en\&amp;nrm =iso\&amp;tlng=pt.

16. Wang S, Xie L, Xu Y, Yao B, Xiang D. Sleep disturbances among medical workers during the outbreak of COVID-2019.Wuhan: Occupational Medicine [Internet] 2020 [acesso em 4 de Agosto 2020]; 70 (6), 364-369. Disponível em: https://www.researchgate.net/publicati on/341216548_Sleep_disturbances_am ong_medical_workers_during_the_out break_of_COVID-2019.

17. Ornell F, Halpern SC, Kessler FHP, Narvaez JCM. The impact of the COVID-19 pandemic on the mental health of healthcare professionals . Porto Alegre : Cad. de Saúde Pública, [Internet] 2020[acesso em 4 de Agosto 2020] ; 36(4) ,1-6 , 2020. Disponível em: http://dx.doi.org/10.1590/0102$311 \times 00063520$.

18. Vanni, Gianluca et al. The Effect of Coronavirus (COVID-19) on Breast Cancer Teamwork: a multicentric survey. Italia: In Vivo, [Internet] 2020 [acesso em 4 de Agosto 2020];34(3), 1685-169. Anticancer Research USA Inc.

http://dx.doi.org/10.21873/invivo.1196 2.

19. Zhang SX, Liu J, Jahanshahi AA, Nawaser K, Yousefi A, Jizhen L, et al. At the height of the storm: Healthcare staff's health conditions and job satisfaction and their associated predictors during the epidemic peak of COVID-19. China: Rain Behavior and Immunity [Internet] 2020 [acesso em 4 de Agosto 2020]; 87 (2020) 144-146. Disponível em: https://pesquisa.bvsalud.org/gim/resour ce/en/mdl-32387345. 
20. Zerbini G, Egigbo A, Reicherts P, Kunz M, Messman H. Psychosocial burden of healthcare professionals in times of COVID-19 - a survey conducted at the University Hospital Augsburg. Alemanha: German Medical Science [Internet] 2020 [acesso em 4 de Agosto 2020]; 18. Disponível em: https://pesquisa.bvsalud.org/portal/reso urce/es/mdl-32595421.

21. Zhang Wr, Wang K, Yin L, Zhao WF, Xue Q, Peng M. Mental Health and Psychosocial Problems of Medical Health Workers during the COVID-19 Epidemic in China. China: Psychother Psychosom. [Internet] 2020 [acesso em 4 de Agosto 2020]; 89, 242-250. Disponivel em: https://pesquisa.bvsalud.org/globalliterature-on-novel-coronavirus-2019ncov/resource/en/covidwho-305902

22. Sasangohar, Farzan; JONES, Stephen L.; Masud, Faisal N.; Vahidy, Farhaan S.; Kash, Bita A. Provider Burnout and Fatigue During the COVID-19 Pandemic: lessons learned from a highvolume intensive care unit. Texas: Anesthesia \& Analgesia,[Internet] 131(1), 106-111. Ovid Technologies (Wolters Kluwer Health). http://dx.doi.org/10.1213/ane.0000000 000004866.

23. Yin Q, Sun Z, Liu T, Ni X, Deng X, Jia $\mathrm{Y}$, et al. Posttraumatic stress symptoms of health care workers during the corona virus disease 2019. China: Clin Psychol Psychother [Internet] 2020 [acesso em 4 de Agosto 2020]; 27, 384-395. Disponível em: https://europepmc.org/article/med/3241 5733.

24. Sun D, Yang D, Li Y, Zhou J, Wang W, Wang Q .Psychological impact of 2019 novel coronavirus (2019-nCoV) outbreak in health workers in China. China: Epidemiology and Infection [Internet]2020[acesso em 4 de Agosto 2020]; 148(96) , 1-6.Disponível em: https://www.cambridge.org/core/journa ls/epidemiology-and-

infection/article/psychological-impactof-2019-novel-coronavirus-2019ncovoutbreak-in-health-workers-inchina/713EE65B2FB9E9F1B8C0B4C A72F3E87A.

25. Temsah MH, Al-Sohime F, Alamro N, Al-Edyadh A, Al-Hasan K, Jamal A. The psychological impact of COVID19 pandemic on health care workers in a MERS-CoV endemic country. Arábia Saudita: Rev de infecção e saúde pública [Internet] 2020 [acesso em 4 de Agosto 2020]; 13, 877-882. Disponível em:

https://covid19.elsevierpure.com/ca/pu blications/the-psychological-impact-ofcovid-19-pandemic-on-health-carework. 
26. Anmella G, Fico G, Roca A, GómezRamiro M, Vázquez M, Murru A, et al. Unravelling potential severe psychiatric repercussions on healthcare professionals during the COVID-19 crisis. Journal of Affective Disorders [Internet] 2020 [acesso em 4 de Agosto 2020]; 273, 422-424. Disponível em: https://covid19.elsevierpure.com/de/pu blications/unravelling-potential-severepsychiatric-repercussions-on-healthc.

27. Rossi, Rodolfo; Socci, Valentina; Pacitti, Francesca; Lorenzo, Giorgio di; Marco, Antinisca di; Siracusano, Alberto; Rossi, Alessandro. Mental Health Outcomes Among Frontline and Second-Line Health Care Workers During the Coronavirus Disease 2019 (COVID-19) Pandemic in Italy. Jama Network Open [Internet] 2020 [acesso em 4 de Agosto 2020] 3 (5) , 1-4. Disponível

m:http://dx.doi.org/10.1001/jamanetwo rkopen.2020.10185.

28. Peter E, Wu MD, Syrtra MD, Wayne L, Gold MD. Mitigating the psychological effects of COVID-19 on health care workers. Toronto:Mental Health [internet] 2020 [acesso em 4 de Agosto 2020]; 192 (18). Disponível em:

https://pesquisa.bvsalud.org/globalliterature-on-novel-coronavirus-2019ncov/resource/en/covidwho-66415.
29. Rolim Neto ML, Almeida HG, Esmeraldo JD, Nobre CB, Pinheiro WR, Oliveira CRT, et al. When health professionals look death in the eye: the mental health of professionals who deal daily with the 2019 coronavirus outbreak. Psychiatry Research [Internet]. 2020 [acessoem12 mai 2020]; 288, 112972-112981. Disponível em: https://www.ncbi.nlm.nih.gov/pmc/arti cles/PMC7152886/

30. Khan S, Siddique R, Ali A, Bai Q, Li $\mathrm{Z}$, Li H, et al. The spread of novel coronavirus has created an alarming situation worldwide. Journal Of Infection And Public Health [Internet].2020 [acesso em14 mai 2020]; 13 (4): 469-471. Disponível em:

https://www.ncbi.nlm.nih.gov/pmc/arti cles/PMC7129132/

31. Grincenkov FR. A Psicologia Hospitalar e da Saúde no enfrentamento do coronavírus: necessidade e proposta de atuação. HU Revista [Internet]. 2020 [acesso em18 mai 2020]; 46, (3): 3-4. Disponível em: https://periodicos.ufjf.br/index.php/hur evista/article/view/30050

32. Filho JMJ, Assunção AA, Algranti E, Saito CA, Maeno M. A saúde do trabalhador e o enfrentamento da COVID-19. Revista Brasileira de 
Saúde Ocupacional [Internet]. 2020 [acesso em 18 mai 2020];45, 1-3. Disponível em: https://www.scielo.br/scielo.php?script =sci_arttext\&pid=S030376572020000100100.

33. Wu K, Wei $\mathrm{X}$. Analysis of psychological and sleep status and exercise rehabilitation of front-line clinical staff in the fight against COVID-19 in China. Medical Science Monitor Basic Research [Internet]. 2020 [acesso em 06 ago 2020]; 26 (e924085): e924085-1 - e924085-7. Disponível em: https://www.researchgate.net/publicati on/341296191_Analysis_of_Psycholo gical_and

Sleep_Status_and_Exercise_Rehabilit ation_of_FrontLine_Clinical_Staff_in _the_Fight_Against_COVID19_in_China

34. Schmidt B, Crepaldi MA, Bolze SDA, Neiva-Silva L, Demench LM. Impactos na Saúde Mental e Intervenções Psicológicas Diante da Pandemia do Novo Coronavírus (COVID-19). Estudos de Psicologia (Campinas) [Internet]. 2020 [acesso em 18 mai 2020]; 1-26. Disponível em:

https://www.scielo.br/scielo.php?scri $\mathrm{pt}=$ sci_arttext\&pid=S0103166X2020000100501.
35. Makino M, Nakajima AKA, Takebayashi Y. Mental health crisis of Japanese health care workers under COVID-19.American Psychological Association [Internet]. 2020 [acesso em 05 ago 2020]; 12 (S1): S136-S137. Disponível em: https://doi.apa.org/fulltext/2020-44057001.pdf.

36. Agudelo HAM, P. Sacks S, Almondes KM, Pérez GJT, Liparoti P, Rey MJM. Enfrentamento psicológico do COVID 19, documento consenso. Federación latinoamericana de sociedades de sueño e asociación latinoamericana de psicologia del sueño [Internet]. 2020 [acesso em 22 mai 2020]. Disponível em:

https://sochimes.cl/Consenso_COVID -19.pdf

37. Lima CKT, Lima IAAS, Nunes JVAO, Saraiva JS, Souza RI, Silva GL, Neto MSR, et al. The emotional impact of Coronavirus 2019-nCoV (new Coronavirus disease). Psychiatry Research [Internet]. 2020 [acesso em 15 mai2020 ]; 287, 112915-112916. Disponível em: https://www.unboundmedicine.com/me dline/citation/32199182/The_emotional _impact_of_Coronavirus_2019nCoV_(new_Coronavirus_disease).

38. Greenberg N, Docherty M, Gnanapragasam S, Wessely S, et al. 
Managing mental health challenges faced by healthcare workers during covid-19 pandemic. Bmj [Internet]. 2020 [acessoem14 mai 2020]; 1-4. Disponível em: https://www.bmj.com/content/368/bmj .m1211

39. Cunha QB, Camponogara S, Freitas EO, Pinno C, Dias GL, Cesar MP. Fatores que interferem na adesão as precauções padrão por profissionais da saúde: Revisão Integrativa. Enferm. Foco [Internet]. 2017 [acesso em: 22 mai 2020 ]; 8 (1): 72-76. Disponível em:

http://revista.cofen.gov.br/index.php/e nfermagem/article/view/980/358

40. Saidel MGB, Lima MHM, Campos CJG, Loyola CMD, Esperidião E, Rodrigues J. Intervenções em saúde mental para profissionais de saúde frente a pandemia de coronavírus. Rev
Enfermagem UERJ [Internet]. 2020 [acesso em 23 mai 2020]; 28:1-6. Disponível em: https://www.epublicacoes.uerj.br/index.php/enferma gemuerj/article/view/49923/33859

41. Miranda FMDA, Santana LL, Pizzolato AC, Saquis LMM. Condições de trabalho e o impacto na saúde dos profissionais de enfermagem frente a covid-19. Cogitare enferm [Internet]. 2020 [acesso em 23 mai 2020]; 25. Disponível em: https://revistas.ufpr.br/cogitare/article/v iew/72702/pdf

42. Shuja KH, Aqeel M, Jaffar A, Ahmed a. Covid-19 pandemic and impending global mental health implications. Psychiatria Danubina [Internet]. 2020 [acesso em22 mai 2020];32(1):32-35. Disponível em: http://www.psychiatriadanubina.com/UserDocsImages/pdf/d nb_vol32_no1/dnb_vol32_no1_32.pdf 